\title{
ARCHITECTURE OF AN AUTOMATED AGRICULTURAL TRACTOR: HARDWARE, SOFTWARE AND CONTROL SYSTEMS
}

\author{
Ray Eaton, Jayantha Katupitiya*, Anthony Cole and Craig Meyer*
}

\author{
School of Electrical Engineering and Telecommunications \\ * School of Mechanical and Manufacturing Engineering \\ University of New South Wales \\ Sydney, Australia \\ R.Eaton@unsw.edu.au
}

\begin{abstract}
Using a compact agricultural tractor, an automated mobile platform is being developed to support precise agricultural tasks. The task being considered is fruit picking, however the platform developed should be generic. The need for precise control of the tractor, loader mechanism and the task implement attached to the tractor, is paramount. Development of the automated mobile platform can be broken down into various components, namely, the steering, loader, and traction control systems, and safety system. Development for each system includes modifications to hardware, and design of appropriate software to drive the systems automatically. Much work is needed to provide the expert control for the types of precision tasks envisaged. Preliminary work shows simple control of the steering and loader systems. Copyright (C)2005 IFAC
\end{abstract}

Keywords: Agriculture, autonomous vehicles, cascade control, vehicle dynamics, traction control.

\section{INTRODUCTION}

Agricultural automation is an area that has existed in various forms for several decades, but is in increasing demand now. To date, most progress in the area has benefited well structured agricultural tasks. Typical examples of such automated structured tasks include harvesting wheat and cotton crops, and greenhouse based pesticide spraying.

Regardless of the task, most automated systems comprise a mobile element such as a tractor, and a manipulative element such as a back-hoe or robot arm. Depending on the type of task and system design approach, the level of cooperation between the elements and the resulting precision, varies. For example, in the broad acre cropping system described in (Pilarski et al., July 2002), emphasis is given to nav-

\footnotetext{
1 Research partially supported by ARC Centre for Autonomous Systems and the State Government of New South Wales, Australia
}

igation of the mobile vehicle, and not to any coordinated control between it and the manipulative element. In contrast, coordinated control between the mobile and manipulative element is required for the greenhouse harvesting system described in (Henten et al., Nov 2002), and the mechanical weeding system of (Astrand and Baerveldt, July 2002). A class of structured systems include those that are labelled "auto-steer" systems. These are guided systems that exploit existing structure in their environment, such as a row, or predefined pattern of crops. This is a common class of systems, of which (Gerrish et al., 1997), (Stombaugh et al., 1998), (Thuilot et al., July 2002), (Reid, 1998a), and (Elkaim et al., 1996) are examples. Also, (Reid, 1998b) presents an interesting review of the recent status in the area.

The task of controlling the mobile element is a challenging one. As well as requiring precision, the controller has to contend with uncertainty arising from 
within the tractor's working environment as well as changes in its structure. Ground conditions may be uncertain due to loose soil and may be undulating, and the workspace may contain obstacles such as trees, or animals and humans. For the tractor itself, as suggested in (Elkaim et al., 1996), the possibility of different manipulative elements gives rise to varying tractor dynamics. Further, the very movement of the loader mechanism results in time-varying and nonlinear tractor dynamics, particularly if the loader and implement exhibit non-smooth motion. Other nonlinear effects arise from the hydraulic actuator circuits driving the various subsystems.

Due to the scope of the problem, work in the area of generic tractor control often focuses on a smaller subset of problems. For example, bodies of work, (Peng et al., 1997), and (Adams, 2002) consider "lower" level issues such as suspension and tyre damping and their effect. Models are constructed and used for simulation. In (Adams, 2002), such issues are applied to agricultural vehicles specifically, with the author discussing variations of tyre models, and the effect on ride performance. On a "higher" level, much work has been undertaken in areas such as path planning and obstacle avoidance, where little or no emphasis is placed on the lower level issues. Other work exists of course, somewhere in between, where the research addresses smaller, or specific issues. Work in (Elkaim et $a l ., 1996)$ includes the identification and robust control of a tractor for guided (straight line and curved) and constant speed tasks. The identified kinematic model applied the tractor speed and steering wheel angle as inputs, where no emphasis is placed on the underlying dynamics governing the speed and steering control.

For the system currently under development, research efforts are focused on obtaining precise control of the mobile tractor element. This includes the coordinated control of steering, traction and the loader position. The precise control will be motivated by the specific task carried out by the manipulative element. It should also be developed such that it is generic, and can be applied for a range of agricultural tasks such as fruit picking, but also other structured and unstructured tasks. This paper presents the preliminary work undertaken in the development of the mobile element, including the hardware and software development for its automation, as well as some early, yet promising results from the independent control of tractor subsystems.

\section{THE INTEGRATED SYSTEM}

Fig. 1 shows the integrated system, including a 6-DOF articulated robot arm used as the fruit picking manipulative element. The robot is mounted on a telescopic translation stage consisting of an upper and a lower stage, allowing the robot arm to traverse the entire width of the tractor as well as beyond. The loader

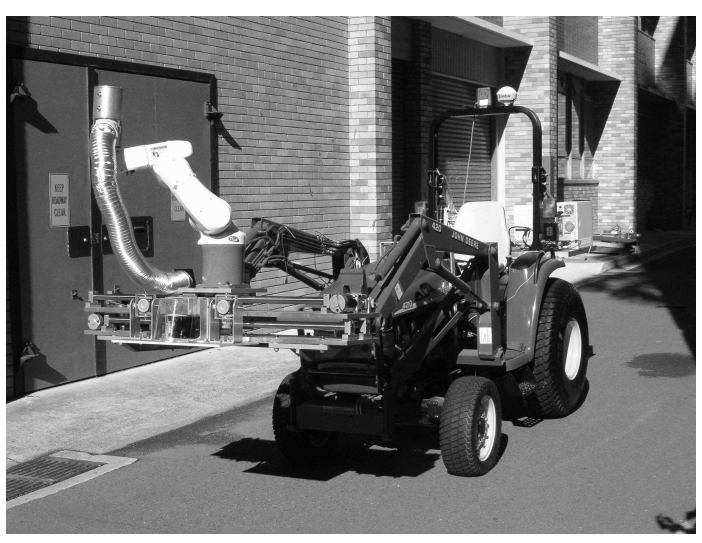

Fig. 1. The integrated system

mechanism carrying the robot arm is hydraulically driven, with two degrees of freedom. This allows the robot arm and translational stage to be elevated to a desired height with a desired tilt angle. Navigation and obstacle avoidance is to be achieved through the use of a laser range finder, stereo cameras, and a GPS system. Communication and data logging is maintained via a wireless Ethernet link to a remote command station.

For control, the tractor systems of interest include the steering, traction, and loader systems. All are governed by hydraulic circuits. The steering system is driven by its own hydraulic pump in an open center architecture. The traction, or hydrostatic transmission, system forms the second hydraulic circuit. As this system works without a tank, a pump will make the oil flow in either direction, thereby driving a hydraulic motor resulting in tractor motion. The third hydraulic circuit is for the implements, which for this tractor, includes the loader and the rockshaft, despite the later not being used for current research. All three hydraulic circuits play vital roles in the automation procedures discussed in the sections to follow.

\section{THE CONTROL ARCHITECTURE}

Broadly speaking, the aim of the tractor controller is to achieve precise control of the tractor plus loader mechanism for generic agricultural tasks. Specifically, it needs to ensure sufficiently precise tracking of a desired tractor state, comprising tractor position, speed, orientation, and loader height and tilt angle. The tractor control system can be described by the block diagram of Fig 2.

The block diagram shows a cascade structure. The inner loop provides control of the various tractor sub-systems variables, including steering wheel angle, tractor speed (traction), and the loader height and tilt angle. The outer loop provides higher level control, determining the required sub-system setpoints from desired and actual tractor system state. Actuation of the entire tractor plus loader occurs via the sub-system hydraulic circuits for steering, traction and the loader. Any coupling between the sub-systems is assumed to 
occur implicitly within the sub-system blocks. Both the tractor sub-systems, and the higher level system are perturbed by various disturbances, such as environmental uncertainty. In reference to the block diagram, work thus far has been focused on modeling of some of the tractor sub-systems and the implementation of the inner sub-system control loop.

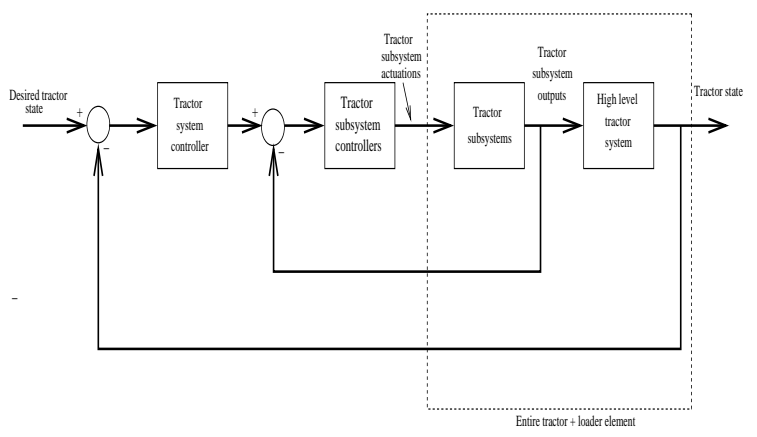

Fig. 2. The tractor control system

\section{STEERING CONTROL SYSTEM}

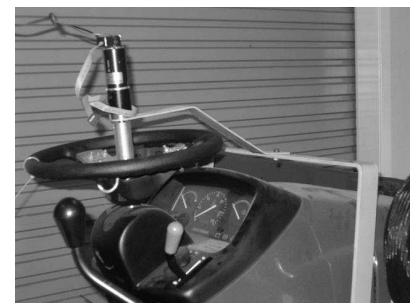

Fig. 3. Steering motor

A hydraulic circuit drives the steering system. To control the steering system, it is necessary to sense the position of the steered road wheels. This is done directly by measuring the displacement of the power cylinder of the steering system, using a linear position transducer. This displacement is mechanically coupled to the steered angles of the front wheels. Measuring the steering wheel position is not sufficient, as this will not necessarily reflect the true position of the steered road wheels. Actuating the steering is achieved by attaching a permanent magnet DC motor to turn the steering. This arrangement is shown in Fig 3.

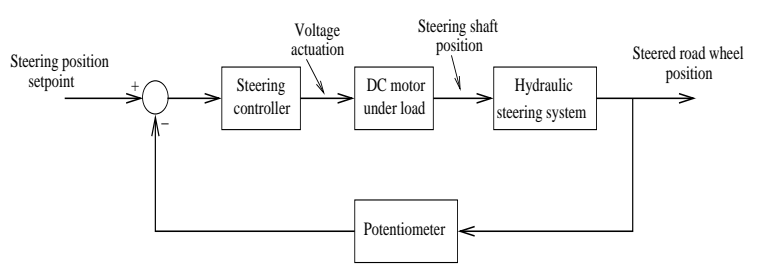

Fig. 4. Steering control system

The steering control system is depicted in the block diagram of Fig4. A series of small steps in both directions, applied to the steering input in open loop, enabled a preliminary, but indicative dynamic model of the steering system to be determined. Upon analysis, a third order linear model was chosen. For steering angle $\delta$, and DC motor input voltage $V_{s}$, the model transfer function is given by (1). This model gives rise to a dominant time constant of approximately 1.05 seconds.

$$
\frac{\delta(s)}{V_{s}(s)}=\frac{94}{s\left(s^{2}+4.5 s+4.5\right)}
$$

Building up a complete and accurate dynamic model of the tractor and its sub-systems will be an important task, particularly in light of the precision control required. It should be noted that although the modelling exercise undertaken provides some insight and indication of the steering system dynamics, it is certainly not a comprehensive analysis. Testing was done with small steps only, mostly around the steering centre, and with the single condition of zero tractor speed. Coupled with that, the data obtained was indicative of non-linear behaviour, not represented by the linear model.

The steering feedback loop was closed by implementing simple PI control. The gains were chosen to yield the response in Fig 5. This represents a compromise between speed of response and steering overshoot. The type of response achieved under feedback control will be important as it may have a significant effect on the overall tractor dynamics. The control achieved here is only preliminary and indicative, and it is still early in development to claim success in this regard. However the response shows reasonably "tight" control, particularly for a negative step. The oscillation observed for the positive step is a further indication of non-linear effects.

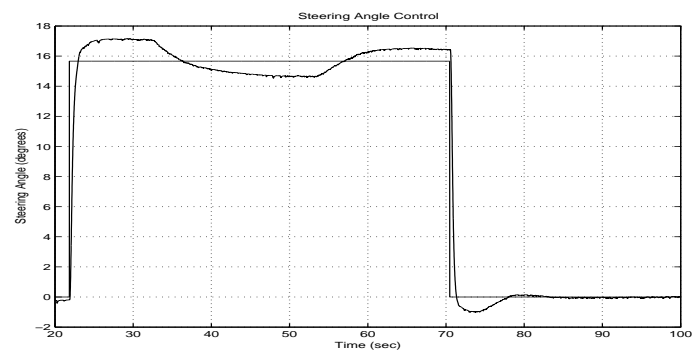

Fig. 5. Closed loop steering control: $K_{p}=1.4$, $T_{i}=2.5$

\section{TRACTION CONTROL SYSTEM}

The forward and reverse motion of the tractor is governed by a hydrostatic transmission system, which is in turn controlled by the tractor's built-in computer. In manual operation, separate pedals, each connected to a potentiometer, control forward and reverse motion. Each potentiometer must provide a voltage in the range $0.7 \mathrm{~V}$ (minimum speed) to $4.23 \mathrm{~V}$ (maximum speed) which is input to the built-in computer, for driving the transmission system. Only one pedal is allowed to operate at any time in the specified range, else an error condition will arise. For automatic operation of 
the traction system, two voltages are generated from the interface card, multiplexed with, and mimicking, the pedal voltages.

For sensing tractor wheel speed, a variable frequency pulse train is provided, giving an indication of the speed of rotation of the mid point of the rear axel. The higher the speed, the larger the frequency of the pulse train. To convert the digital pulse train signal into a signal representing speed, the event counter mechanism will be utilised on the data acquisition card. As with the steering control system, it is intended to feed back the speed signal to provide closed loop speed and position control, however currently, with the pulse train signal not interfaced, all work is undertaken with constant tractor speed in open loop.

\section{LOADER CONTROL SYSTEM}

The loader system is a hydraulically actuated attachment to the front of the tractor. Computer control of the loader in conjunction with the tractor itself is an additional and challenging problem. As discussed in Section 2, the loader has two degrees of freedom, and is responsible for carrying the translational stage which in turn carries a robot arm. The first degree of freedom is for lifting and lowering, while the second is for tilting the front end of the loader mechanism. Each degree of freedom of the loader has its own actuating cylinder and a flow control valve.

In manual operation, the two degrees of freedom are controlled via a selective control valve (SCV). To automate the loader operation, a separate hydraulic circuit was designed with two pairs (one for each degree of freedom) electrically controlled valves (ECV) that operate in parallel with the existing SCV, thus ensuring both manual operation and computer controlled operation can co-exist.

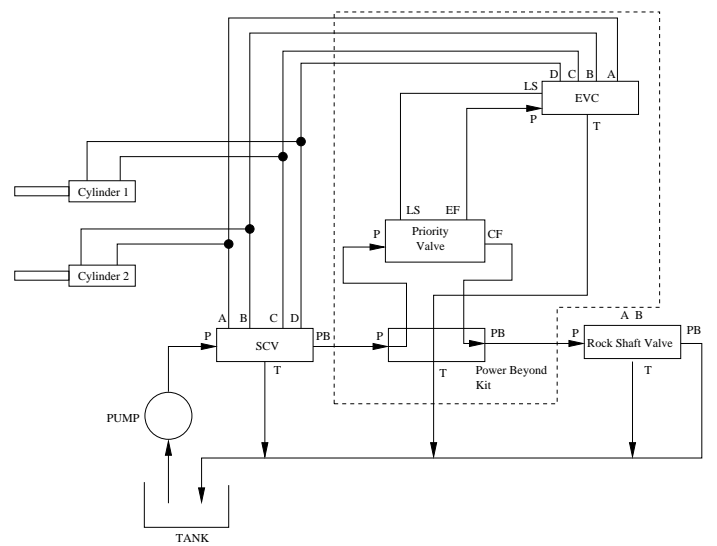

Fig. 6. Schematic of loader hydraulic circuit

A schematic of the designed hydraulic system is shown in Fig. 6. The area demarcated by the dotted line in Fig. 6 shows the modifications carried out to the hydraulic system. The original hydraulic circuit is of open centre type. Modifications include the addition of a power beyond kit, used to provide an access point for another open centre valve, the ECV, providing identical parts for cylinder 1 and 2, and the priority valve block, introduced to account for the closed centre valve, ECV, being present in an open centre system. A more detailed explanation is provided in (Katupitiya et al., 2005).

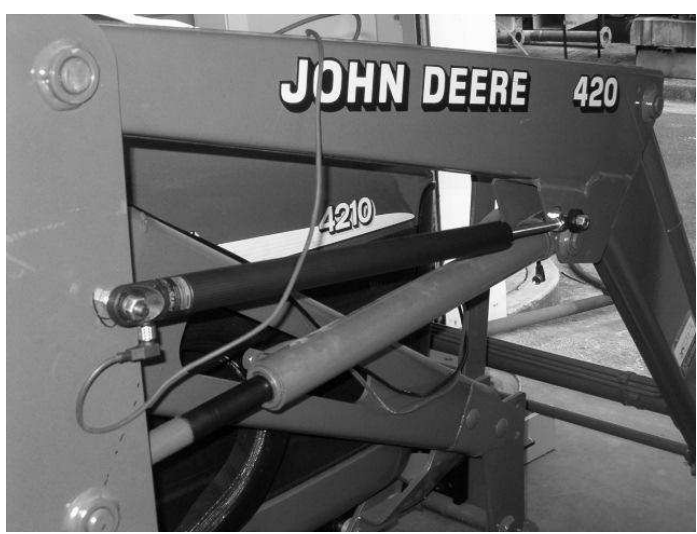

Fig. 7. Loader position sensor

Feedback control of the loader sub-system minimally requires measurement of the loader height and tilt angle. This is achieved via the use of linear potentiometers, as shown in Fig. 7. Rather than measure the height and tilt angle directly, the sensors give an indication of the loader angles with respect to the fixed tractor frame. Inverse kinematics is then used, such that for a desired loader height and tilt angle, the necessary loader angles, and hence linear potentiometer displacements can be computed. Of course, effects such as tractor tilt and ground undulation, will have an effect on the loader variables, however this can be dealt with by the high level tractor control, which will make use of the additional sensor feedback.

The block diagram of Fig 8 describes the loader control system, controlling both loader height and tilt angle. Independent control loops are used to control each degree of freedom, and coordination is required if the tilt angle is to be specified with respect to the fixed tractor frame, or ground, rather than with respect to the loader arm. For example, when using the fruit-picking robot, the tilt angle should be regulated to zero with respect to the ground regardless of the loader height.

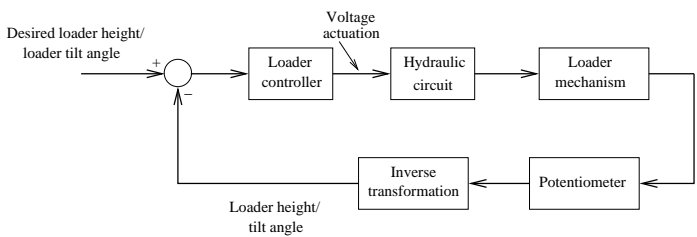

Fig. 8. Loader control system

As was the case with the steering control system, a series of small steps in both directions were applied in open loop, to ascertain some model information about the load mechanism. At this stage, only loader height is considered. Once again, analysis lead to a third 
order model, with a dominant time constant of approximately $0.42 \mathrm{sec}$. For loader height above ground, $h$, and loader actuation voltage, $V_{l}$, the following model, (2), is obtained

$$
\frac{h(s)}{V_{l}(s)}=\frac{5.62}{s\left(s^{2}+8.5 s+15\right)}
$$

As with the steering sub-system, the modelling exercise undertaken provides some insight and indication of the loader system dynamics, but is not a comprehensive analysis. In fact, further data obtained was also indicative of non-linear behaviour, with dynamics depending on the operating region.

Simple closed loop PI control was then implemented to yield the response in Fig 9. This preliminary control scheme was chosen to also achieve a compromise between speed of response and loader height overshoot.



Fig. 9. Closed loop loader control: $K_{p}=1.5$, $T_{i}=2.0$

\section{THE WATCHDOG SYSTEM}

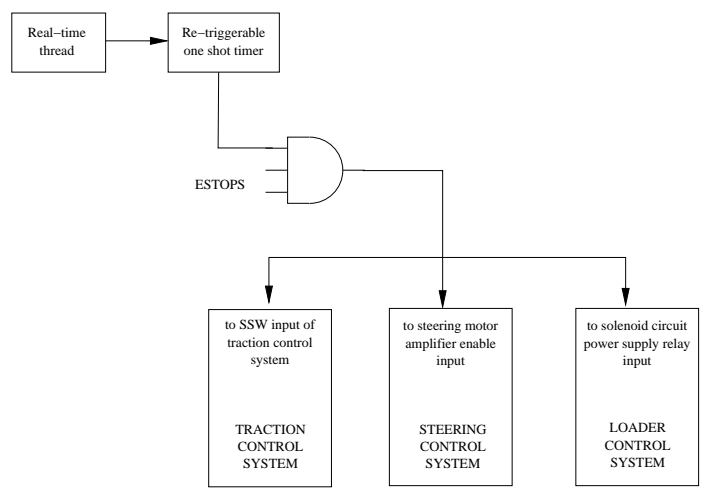

Fig. 10. Watchdog system

A vital part of the tractor system is the watchdog system, responsible for ensuring safe operation of the tractor under all circumstances. In the event of any fault condition, the watchdog system is required to halt all mechanical subsystems of the tractor. The effect of the watchdog system output must be identical to a manually operated emergency stop. Thus all manually operated emergency stops (ESTOP) must be wired OR with the watchdog system output. Each of the tractor subsystems, namely, the traction control system, steering control system, and loader control system, implement enable inputs that are used to enable/disable each system independently. As software forms an integral part of the system, detecting the software failures is of paramount importance. Software interacts with the watchdog circuitry, by re-triggering a one-shot timer periodically. This re-triggering keeps the watchdog system alive. Faliure to re-trigger the timer will result in a halt in tractor operations. Fig. 10 shows a schematic of the watchdog signal generation. As shown in the figure, to halt the traction control system, the seat switch signal (SSW) is used. The seat switch indicates the presence of a driver at the seat. The seat switch must be activated for tractor motion to be enabled. On the assumption that the manufacturer's safety subsystem has a high degree of reliability, the seat switch (SSW) forms an ideal enable signal for the traction control system.

The risks associated with the steering control system are much less threatening than those of the traction control system. The enable signal of the amplifier that drives the steering motor is chosen as the enable input for the steering subsystem.

For the loader system, a relay input is used as the enable signal for the loader control system. The relay input cut power to the solenoid drive of the proportional valves.

In the actual implementation, the watchdog signal is multiplexed with the seat switch signal. When the tractor is to be driven by a driver, the actual seat switch signal is connected to the traction control system, thereby allowing a driver to manually operate the tractor. When in automatic mode, the signal coming from the driver seat is replaced by that generated by the watchdog system. Note that the actual seat switch signal is not connected to the steering control system and the loader control system. Thus when the system is in manual mode, the watchdog system or the seat switch has no influence on the steering or loader control systems.

\section{TRACTOR AUTOMATION SOFTWARE}

The software system driving the tractor element is made up of a remote and external on-board computer. The remote computer is Windows driven, and set up with remote wireless access to the on-board computer. Most software tasks however, are implemented on the on-board computer, driven by Linux with a real-time kernel, RTLinux, installed, and in operation for hard real-time performance.

The responsibilities of the software system can be categorised as follows; (i) to provide run-time fault detection and recovery mechanisms on both computers for safe operation; (ii) to provide remote access between the on-board and remote computer platforms; (iii) to provide hard real-time control of the various tractor subsystems; (iv) to allow user interaction with 
the tractor sub-systems to vary system parameters, as well as command the watchdog system; (v) to facilitate remote command and data monitoring, as well as system diagnostics. Several software processes or tasks, executing on both the remote and on-board computer, undertake these responsibilities.

The remote computer is responsible for providing remote command, monitoring, and fault detection capabilities. The on-board computer on the other hand, is interfaced directly to instrumentation devices via the real-time thread, which allow driving and monitoring of the various tractor sub-systems in real-time. It also provides command control and monitoring software capable of being locally and remotely driven, which is useful for development and testing. It too provides fault detection.

The software for controlling the three sub-systems, namely, steering, traction and loader systems, is contained within the real-time thread on the on-board computer. Importantly, it is also responsible for periodically refreshing the one-shot watchdog timer. Failure to reset the timer will result in the watchdog enable signal being negated. In normal operation, the realtime thread will receive fault test information from the on-board fault detection program. Absence of such information is indicative of software failure elsewhere, whether it be on-board or on the remote computer, resulting in the real-time thread halting the tractor's operation and suspending execution.

\section{CONCLUSION}

Research to date, has focused on providing the hardware and software infrastructure for an automated mobile tractor used for precise agricultural tasks. With this infrastructure in place, there is means and motivation to carry out further research to execute such precision tasks. The paper describes the various tractor sub-systems, including the steering, traction, and loader control systems, as well as the watchdog and software system. Preliminary and simple PI control is implemented for the steering and loader subsystems, yielding crude, yet promising results. The addition of the loader implement to the tractor provides an interesting and novel facet to the research, due to the increased complexity of the system, and the need to control both accurately, so that the manipulative element attached to the loader can achieve its objective. Further development of the sub-system controllers will require consideration of their interaction between each other as well as within the tractor as a complete system. For possible avenues of further development, the tractor and its sub-systems may lend itself to a decentralised control approach, or a multivariable design.

Another advantage of the automation design is in the integration of the automation hardware and software with the existing tractor subsystems, as opposed to the replacement of the existing subsystems. This allows the preservation of a manual operation mode. An emphasis is placed on safe operation, giving rise to a robust watchdog system, implemented in both hardware and software. In the event of a fault condition, the watchdog system will ensure a halt in the operation of the tractor.

\section{REFERENCES}

Adams, B. T. (2002). Central tire inflation for agricultural vehicles. $\mathrm{PhD}$ thesis. University of Illinois at Urbana-Champaign.

Astrand, B. and A. J. Baerveldt (July 2002). An agricultural mobile robot with vision-based perception for mechanical weed control. Autonomous Robots 13, Issue 1, 21-35.

Elkaim, G., M. O'Connor, T. Bell and B. Parkinson (1996). System identification and robust control of farm vehicles using cdgps. In: Proceedings of the Institute of Navigation ION-GPS Conference. Kansas City, MO. pp. 485-494.

Gerrish, J. B., B. W. Fehr, G. R. Van Ee and D. P. Welch (1997). Self-steering tractor guided by computer vision. Applied Engineering in Agriculture 13, No. 5, 559-563.

Henten, E. J. Van, J. Hemming, B. A. J. Van Tuijl, J. G. Kornet, J. Meuleman, J. Bontsema and E. A. Van Os (Nov 2002). An autonomous robot for harvesting cucumbers in greenhouses. Autonomous Robots 13, Issue 3, 241-258.

Katupitiya, J., R. Eaton, G. Rodnay, A. Cole and C. Meyer (2005). Automation of an agricultural tractor for fruit picking. To appear, International Conference on Robotics and Automation, May 2005.

Peng, H., R. Strathearn and A. Galip Ulsoy (1997). A novel active suspension design technique - simulation and experimental results. In: Proceedings of 1997 American Control Conference. Albuquerque, New Mexico.

Pilarski, T., M. Happold, H. Pangels, M. Ollis K. Fitzpatrick and A. Stentz (July 2002). The demeter system for automated harvesting. Autonomous Robots 13, Issue 1, 9-20.

Reid, J. F. (1998a). Precision guidance of agricultural vehicles. Document uilu-eng-98-7031.

Reid, J. F. (1998b). A status report on automatic guidance of agricultural vehicles in the us: new frontiers in the 21 st century. In: Document uilu-eng98-7026, The Univeristy of Illinios at UrbanaChampaign. RSJ Meeting, Sapporo, Japan.

Stombaugh, T., E. Benson and J. W. Hummel (1998). Automatic guidance of agricultural vehicles at high speed. Vol. St. Joseph, MI.

Thuilot, B., C. Cariou, P. Martinet and M. Berducat (July 2002). Automatic guidance of a farm tractor relying on a single cp-dgps. Autonomous Robots 13, Issue 1, 53-71. 